\title{
Intramyocellular Lipids, Insulin Resistance, and Functional Performance in Patients with Severe Obstructive Sleep Apnea
}

This article was published in the following Dove Press journal:

Nature and Science of Sleep

\author{
Meng-Yueh Chien ${ }^{1-3}$ \\ Pei-Lin Lee (iD) ${ }^{2-5}$ \\ Chih-Wei $\mathrm{Yu}^{6}$ \\ Shwu Yuan Wei ${ }^{6}$ \\ Tiffany Ting-Fang Shih ${ }^{6}$ \\ 'School and Graduate Institute of \\ Physical Therapy, College of Medicine, \\ National Taiwan University, Taipei, \\ Taiwan; ${ }^{2}$ Center of Sleep Disorder, \\ National Taiwan University Hospital, \\ Taipei, Taiwan; ${ }^{3}$ Center for Obesity, \\ Lifestyle and Metabolic Surgery, National \\ Taiwan University Hospital, Taipei, \\ Taiwan; ${ }^{4}$ Department of Internal \\ Medicine, National Taiwan University \\ Hospital, Taipei, Taiwan; ${ }^{5}$ Center for \\ Electronics Technology Integration, \\ National Taiwan University, Taipei, \\ Taiwan; ${ }^{6}$ Department of Medical Imaging \\ and Radiology, Medical College and \\ Hospital, National Taiwan University, \\ Taipei, Taiwan
}

Correspondence: Tiffany Ting-Fang Shih Department of Medical Imaging and Radiology, Medical College and Hospital, National Taiwan University, No. 7, Chung Shan S. Road, Taipei 10002, Taiwan Tel +886223I23456 ext 65568 Email ttfshih@ntu.edu.tw
Purpose: An increasing number of studies have linked the severity of obstructive sleep apnea (OSA) with metabolic dysfunction. However, little is known about the lipid compartments (intramyocellular [IMCL] and extramyocellular [EMCL] lipids) inside the musculature in these patients. The present study was designed to investigate the IMCL and EMCL, biochemical data, and functional performance in patients with severe OSA, and to examine the correlations between intramuscular lipid contents and test variables.

Participants and Methods: Twenty patients with severe OSA (apnea-hypopnea index [AHI]: $\geq 30 / \mathrm{h}$; body mass index [BMI]: 26.05 \pm 2.92 ) and 20 age- and BMI-matched controls $(\mathrm{AHI}<5 / \mathrm{h})$ were enrolled. Proton magnetic resonance spectroscopy was used to measure the IMCL and EMCL of the right vastus lateralis muscle. Biochemical data, including levels of fasting plasma glucose, insulin, lipid profiles, and high-sensitivity C-reactive protein (hsCRP), were measured. Insulin resistance index (IR) was calculated using the homeostasis model assessment method. Performance tests included a cardiopulmonary exercise test and knee extension strength and endurance measurements.

Results: Patients with severe OSA had significantly $(P<0.05)$ lower values of IMCL $(14.1$ $\pm 5.4 \mathrm{AU})$ and EMCL (10.3 $\pm 5.8 \mathrm{AU})$ compared to the control group (25.2 $\pm 17.6 \mathrm{AU}$ and 14.3 \pm 11.1 AU, respectively). Patients with severe OSA had significantly higher hsCRP, IR, and dyslipidemia compared with controls (all $P<0.05$ ). Furthermore, IMCL was negatively correlated with AHI, cumulative time with nocturnal pulse oximetric saturation lower than $90 \%\left(\mathrm{TSpO}_{2}<90 \%\right)(\rho=-0.35, P<0.05)$, IR $(\rho=-0.40, P<0.05)$, glucose $(\rho=-0.33, P<0.05)$, and insulin $(\rho=-0.36, P<0.05)$, and positively correlated with lowest oximetric saturation $(\rho=0.33, P<0.01)$.

Conclusion: Skeletal muscle dysfunction and metabolic abnormalities were observed in patients with OSA that did not have obesity. IMCL was positively correlated with aerobic capacity and muscular performance, but negatively correlated with AHI and IR. Large-scale clinical trials are required to explore the complicated mechanism among OSA, intramuscular metabolism, and insulin action.

Clinical Trial Registration: ClinicalTrials.gov Identifier: NCT00813852.

Keywords: ${ }^{1} \mathrm{H}$ magnetic resonance spectroscopy, insulin resistance, obstructive sleep apnea, skeletal muscle

\section{Introduction}

Obstructive sleep apnoea syndrome (OSA) is characterized by repeated occlusion of the upper airway during sleep, resulting in periods of intermittent hypoxemia. ${ }^{1}$ An increasing number of studies have linked the severity of OSA with metabolic 
dysfunction. ${ }^{2,3}$ Particularly, both glucose intolerance and insulin resistance are closely correlated with the level of nocturnal oxygen desaturation in patients with OSA. ${ }^{2}$ As skeletal muscles have the largest tissue mass for the uptake of circulating glucose, it is speculated that altered intramuscular energy metabolism may implicate the development of insulin resistance. ${ }^{4,5}$ Previous studies have reported that oxidative metabolism in the skeletal muscle is compromised in patients with intermittent hypoxia ${ }^{6}$ and in patients with OSA. ${ }^{7,8}$ The increased reliance on anaerobic energy sources and decreased oxidative capacity may thus influence insulin sensitivity. ${ }^{4,5}$ However, little is known about the lipid compartments inside the musculature in patients with OSA.

Besides the subcutaneous fat layer, there are two more lipid compartments located inside the musculature: the extramyocellular (EMCL) and intramyocellular lipids (IMCL). The EMCL nestles in layers along the muscles fiber bundles, whereas the IMCL consists of microscopic, nearly spherical fat droplets within the myocytes, and acts as a fuel source for the myocytes. ${ }^{9}$ Type I fibers contain more IMCL than type II (glycolytic) fibers. ${ }^{10}$ Several studies reported a significant association of increased IMCL with insulin resistance. ${ }^{11} \mathrm{It}$ is believed that in Caucasian people, the deposition of IMCLs represents an early abnormality in the pathogenesis of insulin resistance. However, investigations on IMCL in Indians and South Asian populations failed to show any relationship between IMCL and insulin sensitivity. ${ }^{12,13}$ Additionally, both OSA and insulin resistance are pro-inflammatory conditions. However, to our knowledge, the relationships between IMCL and pro-inflammatory markers, such as highsensitivity C-reactive protein (hsCRP), have not been extensively investigated.

Invasive technique (eg, skeletal muscle biopsy) and noninvasive methods (eg, computed tomography, proton magnetic resonance spectroscopy [ $\left.{ }^{1} \mathrm{H}-\mathrm{MRS}\right]$, and magnetic resonance imaging) can be used to assess IMCL. ${ }^{14}$ The invasive nature of muscle biopsies and the safety issues concerning ionizing radiation from computed tomography limit the determination of the lipid stores. ${ }^{1} \mathrm{H}-\mathrm{MRS}$ is a noninvasive tool allowing the quantification of skeletal muscle lipids, especially for differentiating between IMCL and EMCL. ${ }^{15}$ With skeletal muscle lipids and energy production playing such an influential role in glucose control and lipid oxidation, ${ }^{16}{ }^{1} \mathrm{H}-\mathrm{MRS}$ provides data that will supply information gained from circulating substrates and signaling hormones. Although ${ }^{1} \mathrm{H}-\mathrm{MRS}$ has been extensively used in the investigation of metabolism in other diseases, ${ }^{16}$ only few studies utilized this technique to investigate skeletal muscles' metabolism in patients with OSA. ${ }^{17,18}$

Therefore, the purposes of this study were to (1) investigate the IMCL and EMCL, biochemical data, and functional performance in patients with severe OSA versus controls, and (2) examine the correlations between intramuscular lipid contents and biochemical (insulin, glucose, hsCRP, and lipid profiles) and performance variables (peak oxygen consumption, and strength and endurance of knee extensors).

\section{Methods}

\section{Participants}

Male patients (ranging from 40 to 65 years of age) who were referred to the Center of Sleep Disorder of a teaching hospital for evaluation of sleep apnea were prospectively recruited in this study since September 2007. We have previously reported the details of the recruitment procedures and methods. ${ }^{19,20}$ Briefly, the consecutive participants who were newly diagnosed with severe OSA with whole-night polysomnography (PSG) (apnea-hypopnea index [AHI] of $\geq 30 \mathrm{~h}^{-1}$ ) were recruited. The control group included age( \pm 3 years), weight- $( \pm 3 \mathrm{~kg})$, and height-matched $( \pm 5 \mathrm{~cm})$ subjects without OSA (AHI $<5 \mathrm{~h}^{-1}$ ). Control subjects were patients who were referred to the sleep laboratory for snoring, sleep disturbance, or excessive daytime sleepiness and were confirmed to not have OSA. Exclusion criteria were coronary heart diseases, nervous system diseases, abnormal pulmonary function, morbid obesity, diabetes under insulin management, alcoholism ( $\geq 50$ g per day), and recent infection. Additionally, participants were excluded if they had any missing value(s) for our primary measures. All participants had never been treated for OSA. Biochemistry, MRS, CPET and other tests were all performed by personnel blinded to the OSA status of the patients.

This trial was conducted in accordance with the Declaration of Helsinki. This study was approved by the Institutional Ethics Committee of the National Taiwan University Hospital (www.ClinicalTrials.gov; NCT00813852) and all subjects provided their written informed consent prior to enrolment.

\section{Outcome Measures Diagnosis of OSA}

Whole-night PSG (Embla N7000, Medicare Flaga, Reykjavik, Iceland) was performed in the sleep lab following the protocol described in the previous studies. ${ }^{19,20}$ The 
sleep stages and respiratory events were scored according to the American Academy of Sleep Medicine standard. ${ }^{1}$ In short, apnea was defined by the absence of airflow $\geq 10$ seconds and hypopnea was a $\geq 50 \%$ decrease in airflow $\geq 10$ seconds associated with reduced arterial oxygen saturation of $\geq 3 \%$ or an arousal. The oxygen desaturation index (ODI) was defined by the number of $\geq 4 \%$ arterial oxygen saturation per hour. All of the sleep studies were analyzed by the same investigator to maximize inter- and intra-scorer reliability. The level of daytime sleepiness was assessed using the Epworth sleepiness scale in the morning after nocturnal PSG. Normal values range from 2 to 10 . Scores over 10 indicate daytime sleepiness. ${ }^{21}$

\section{Biochemistry Investigations}

Fasting (overnight) venous blood samples were drawn from the antecubital vein on the next morning of PSG to estimate hsCRP levels, plasma glucose, insulin level, total cholesterol, triglycerides, and high-density lipoprotein cholesterol level. All tests were performed by personnel blinded to the clinical outcomes. hsCRP levels were measured using CRP-Latex (II) immunoturbidimetric assay kit (Denka Seiken, Tokyo, Japan) on a Hitachi 911 immunoannalyzer (Roche Diagnostics, Indianapolis, IN, USA). ${ }^{22}$ The concentrations of glucose, total cholesterol, triglycerides, and high-density lipoprotein cholesterol were assayed according to the previously described methods. ${ }^{23}$ The value of low-density lipoprotein cholesterol was further calculated using the Friedewald formula. ${ }^{24}$ Serum insulin concentration was estimated using the ${ }^{125} \mathrm{I}$-insulin radioimmunoassay kit (Medicorp Inc., Montreal, Canada). The homeostasis model assessment method was applied to calculate insulin resistance by using the following formula: fasting serum insulin $(\mathrm{mu} / 1$ (micro-units $)) \times$ fasting blood glucose $(\mathrm{mmol} / 1) / 22.5^{25}$

\section{Vital Signs and Body Composition Measurements}

Upon arrival to the laboratory, subjects rested in a chair for at least $5 \mathrm{~min}$ before the heart rate and blood pressure were measured. Next, weight and height were measured, and the body mass index (BMI) was calculated. Body composition was then measured using a bioelectrical impedance analyzer (Maltron BioScan 920, Esgender, UK), with $800 \mu \mathrm{A}$ of current at a frequency of $50 \mathrm{kHz}$. The measurement procedure was carried out according to the user's manual. Once the measurements had stabilized, the analyzer calculated the percentage of body fat directly from the equation and displayed the value. Previous studies have demonstrated an excellent test-retest reliability and validity for the method comparable to hydrostatic weighing and dual-energy X-ray absorptiometry. ${ }^{26,27}$

\section{Functional Performance of Knee Extensors}

A Cybex 6000 (Cybex, Division of Lumex Inc., Ronkonkoma, NY, USA) was utilized to measure the strength and the endurance of right knee extensors. Each subject was instructed to perform five maximal isometric knee extension contractions at a $60^{\circ}$ knee flexion angle. Each contraction lasted for 5 seconds, with a resting period of at least 15 seconds between trials. ${ }^{28}$ The mean of five maximal isometric contraction peak torques (Newtonmeter, N-m) for each subject was calculated for data analyses. The endurance test consisted of 30 cycles of alternative knee extension/flexion isokinetic contractions with the speed of $180^{\circ}$ second. The muscular endurance was evaluated by total works (N-m) generated in 30 cycles of contractions as the total area under the torque curve for knee extension movement which would be automatically provided by the Cybex system. ${ }^{28}$

\section{Cardiopulmonary Exercise Test}

Each subject performed a symptom-limited maximal exercise test with continuous electrocardiographic monitoring on a cycle ergometer. All participants were encouraged to exercise until exhaustion. The criteria for termination included at least two of the following conditions: volitional fatigue, heart rate at or near $90 \%$ of the age-predicted maximum, or a respiratory exchange ratio $>1.15 .^{29}$ The graded exercise protocol consisted of three minute stages, starting at 25 watts with a 25 -watt increment at each successive stage, while maintaining a pedalling rate of 50-60 rpm. Respiratory gas exchange measurements, including peak ventilation $\left(\mathrm{V}_{\text {Epeak }}\right)$ and oxygen consumption $\left(\mathrm{VO}_{2 \text { peak }}\right)$, were obtained during exercise using a computer-controlled, breath-by-breath metabolic measurement system (Vmax29 Metabolic Measurement System; SensorMedics, Anaheim, CA, USA). The tester was blinded to the results of the biochemical analyses.

\section{Proton Magnetic Resonance Spectroscopy ('H-MRS)} Localized ${ }^{1} \mathrm{H}$ MRS spectrum of the vastus lateralis were acquired using a 1.5 Tesla whole-body imager (Sonata, Siemens, Erlangen, Germany). During the measurement acquisition procedure, the subjects remained supine, with the vastus lateralis complex of the right thigh positioned within the homogeneous volume of the magnet. Before performing ${ }^{1} \mathrm{H}$ MRS, multi-slice magnetic resonance images in three orthogonal planes of the right thigh 
(above knee) were acquired using a standard spin-echo pulse sequence [echo time $(\mathrm{TE})=15 \mathrm{~ms}$, repetition time $(\mathrm{TR})=520 \mathrm{~ms}, 5 \mathrm{~mm}$ slice thickness; $256 \times 256$ matrix] Then, these scout images were acquired to position the volume of interest. Volumes of interest (typically $15 \times$ $15 \times 25 \mathrm{~mm}^{3}$ ) centered within the vastus lateralis muscle were placed to avoid vascular structures and gross adipose tissue deposits. For volume selection, a single voxel PRESS (Point Resolved Spectroscopy) technique was applied. Chemical shifts were reported using water as the internal standard at $4.7 \mathrm{ppm}$. The IMCL and EMCL peaks were well identified in the longer echo time sequence $(\mathrm{TR}=3000 \mathrm{~ms}, \mathrm{TE}=20 \mathrm{~ms})$. The spectra were analyzed, including amplitude and area under curve. The spectra obtained several chemical components: TMA= trimethylamines (Choline), at $3.2 \mathrm{ppm} ; \mathrm{TCr}=$ total creatine methyl, at 3.0 ppm; EMCL(-CH2), 1.5 ppm; IMCL(CH2), 1.3 ppm; EMCL(-CH3), 1.1 ppm; IMCL(-CH3), 0.9 ppm. Contributions of IMCL and EMCL contents were estimated by de-convolution of the lipid resonance peak (0.8-1.6 ppm). The percentages of IMCL and EMCL, respective to water, were determined by calculating the area under each peak using system software (Java-based MR user interface (jMRUI)). ${ }^{30}$

\section{Data Analyses}

Statistical analyses were performed using the Statistical Software for Social Sciences v.19 for Windows (SPSS Inc., Chicago, IL, USA). Continuous variables were expressed as mean \pm standard deviation, and categorical variables were expressed as numbers and percentages. Between-group comparisons of baseline measurements were examined using the independent Student's $t$-test, Mann-Whitney $U$-test, or a chi-squared test. The differences of exercise test parameters between the two groups were tested using the Student's $t$-test. The associations among the variables were investigated using the Spearman correlation coefficient $(\rho)$. The $\alpha$ value was set at 0.05 .

\section{Results}

\section{Demographic Characteristics and Sleep Examination Results}

Table 1 shows the basic demographic characteristics and sleep examination results of the patients with severe OSA and of the control subjects. The mean age of the study participants was $50.3 \pm 6.1$ years; a majority of these were in the "overweight" category, with a mean BMI of 25.93
Table I Patient Characteristics and Sleep Measurements

\begin{tabular}{|c|c|c|c|}
\hline & $\begin{array}{l}\text { OSA } \\
\text { Group } \\
(n=20)\end{array}$ & $\begin{array}{l}\text { Control } \\
\text { Group } \\
(n=20)\end{array}$ & $P$-value \\
\hline Age (years) & $50.2 \pm 5.6$ & $50.4 \pm 6.7$ & 0.919 \\
\hline Body weight (kg) & $73.8 \pm 7.8$ & $72.4 \pm 7.8$ & 0.581 \\
\hline Body height $(\mathrm{cm})$ & $168.4 \pm 5.3$ & $167.5 \pm 4.2$ & 0.546 \\
\hline Body mass index $\left(\mathrm{kg} / \mathrm{m}^{2}\right)$ & $26.05 \pm 2.92$ & $25.82 \pm 2.76$ & 0.799 \\
\hline Heart rate (bpm) & $77.9 \pm 4.4$ & $76.7 \pm 4.6$ & 0.401 \\
\hline Systolic blood pressure $(\mathrm{mmHg})$ & $128.6 \pm 6.9$ & $120.0 \pm 6.5$ & 0.001 \\
\hline Diastolic blood pressure $(\mathrm{mmHg})$ & $83.5 \pm 7.5$ & $76.7 \pm 6.4$ & 0.003 \\
\hline Epworth sleepiness scale & $11.5 \pm 3.6$ & $8.4 \pm 3.2$ & 0.007 \\
\hline Apnea-hypopnea index (/hr) & $48.0 \pm 18.9$ & $2.4 \pm 1.2$ & $<0.001$ \\
\hline Oxygen desaturation index $(/ \mathrm{hr})$ & $42.5 \pm 21.5$ & $2.2 \pm 1.0$ & $<0.001$ \\
\hline Lowest $\mathrm{SpO}_{2}(\%)$ & $72.2 \pm 8.2$ & $89.5 \pm 2.4$ & $<0.001$ \\
\hline $\mathrm{TSpO}_{2}<90 \%(\%)$ & $18.4 \pm 23.5$ & $0.1 \pm 0.1$ & 0.001 \\
\hline Arousal index $(/ \mathrm{hr})$ & $11.1 \pm 9.2$ & $4.6 \pm 2.5$ & 0.013 \\
\hline Sleep efficiency (\%) & $82.9 \pm 9.6$ & $92.6 \pm 3.8$ & $<0.001$ \\
\hline Energy expenditure (kcal/ $/ \mathrm{kg} /$ day) & $35.20 \pm 2.71$ & $37.31 \pm 5.99$ & 0.159 \\
\hline Hypertension (\%) & $4(20 \%)$ & $3(15 \%)$ & 0.500 \\
\hline Diabetes (\%) & $4(20 \%)$ & $3(15 \%)$ & 0.500 \\
\hline
\end{tabular}

Notes: Values are as group mean $\pm \mathrm{SD}$ or case numbers (percentage). $\mathrm{TSpO}_{2}$ $<90 \%$ is the cumulative time with nocturnal pulse oximetric saturation lower than $90 \%$. A $P$-value less than 0.05 is statistically significant.

$\pm 2.81 \mathrm{~kg} / \mathrm{m}^{2}$. Age and anthropometric characteristics were matched in the two groups. Compared with the control group, patients with severe OSA demonstrated significantly higher values of Epworth sleepiness scale measurements, AHI, ODI, and the cumulative time with nocturnal pulse oximetric saturation lower than $90 \% \quad\left(\mathrm{TSpO}_{2}<90 \%\right)$ $(P<0.05$ for all); the lowest oximetric saturation (lowest $\mathrm{SpO}_{2}$ ), arousal index, sleep efficiency, and blood pressure were observed in the severe OSA group $(P<0.05$ for all). However, both systolic and diastolic blood pressure were considered "normal" based on the most recent American Heart Association recommendations. ${ }^{31}$ Additionally, the numbers of participants with hypertension and diabetes were not significantly different between the two groups $(P>0.05)$. Daily energy expenditure was not significantly different between the two groups $(P>0.05)$.

\section{Outcome Measurement Results}

Table 2 presents the IMCL and EMCL data for the two groups. Significantly lower values for both IMCL and EMCL were observed in the OSA group (10.3 $\pm 5.8 \mathrm{AU}$ (arbitrary unit) and $14.3 \pm 11.1 \mathrm{AU}$, respectively) compared with the control group $(14.1 \pm 5.4 \mathrm{AU}$ and $25.2 \pm 17.6 \mathrm{AU}$, respectively) $(P<0.05)$. However, although the OSA group had a higher ratio of IMCL to EMCL than the control group $(95.0 \pm 64.8 \%$ and $77.6 \pm 36.8 \%)$, the difference was not 
Table 2 Results of Proton Magnetic Resonance Spectroscopy and Biochemistrical Analyses

\begin{tabular}{|c|c|c|c|}
\hline & $\begin{array}{l}\text { OSA } \\
\text { Group } \\
(n=20)\end{array}$ & $\begin{array}{l}\text { Control } \\
\text { Group } \\
(n=20)\end{array}$ & $P$-value \\
\hline IMCL (AU) & $10.3 \pm 5.8$ & $|4| \pm 5.4$. & 0.037 \\
\hline EMCL (AU) & $|4.3 \pm 1| . \mid$ & $25.2 \pm 17.6$ & 0.023 \\
\hline IMCL/EMCL (\%) & $95.0 \pm 64.8$ & $77.6 \pm 36.8$ & 0.304 \\
\hline Body fat (\%) & $22.30 \pm 5.26$ & $21.17 \pm 5.02$ & 0.492 \\
\hline Fat free mass $(\mathrm{kg})$ & $57.85 \pm 5.31$ & $56.02 \pm 4.51$ & 0.249 \\
\hline hsCRP (mg/dL) & $0.23 \pm 0.14$ & $0.12 \pm 0.06$ & 0.039 \\
\hline Insulin resistance index & $2.69 \pm 1.88$ & $1.38 \pm 0.75$ & 0.017 \\
\hline Insulin $(\mu \mathrm{U} / \mathrm{mL})$ & $9.34 \pm 6.01$ & $5.74 \pm 2.42$ & 0.041 \\
\hline Fasting glucose $(\mathrm{mg} / \mathrm{dL})$ & $116.2 \pm 50.5$ & $94.0 \pm 14.6$ & 0.081 \\
\hline \multicolumn{4}{|l|}{ Lipid Profiles } \\
\hline Total cholesterol (mg/dL) & $205.0 \pm 42.5$ & $186.3 \pm 37.1$ & 0.113 \\
\hline Triglyceride (mg/dL) & $149.9 \pm 89.7$ & $105.6 \pm 51.4$ & 0.132 \\
\hline High-density lipoprotein (mg/dL) & $42.1 \pm 7.0$ & $41.6 \pm 7.1$ & 0.730 \\
\hline Low-density lipoprotein (mg/dL) & $138.7 \pm 37.0$ & $|25| \pm 29.8$. & 0.277 \\
\hline $\mathrm{VO}_{2 \text { peak }}(\mathrm{mL} / \mathrm{kg} / \mathrm{min})$ & $25.68 \pm 3.57$ & $27.84 \pm 2.74$ & 0.038 \\
\hline $\mathrm{HR}_{\text {peak }}(\mathrm{bpm})$ & $|5| .0 \pm 15.6$ & $169.6 \pm 13.9$ & $<0.001$ \\
\hline Knee extensors peak torque $(\mathrm{N}-\mathrm{m})$ & $135.2 \pm 19.2$ & $158.2 \pm 13.0$ & $<0.001$ \\
\hline Knee extensors endurance $(\mathrm{N}-\mathrm{m})$ & $44.03 \pm 15.3$ & $60.1 \pm 20.5$ & 0.005 \\
\hline
\end{tabular}

Notes: Values are as group mean \pm SD. A P-value less than 0.05 is statistically significant.

Abbreviations: IMCL, intramyocellular lipid contents; EMCL, extramyocellular lipid contents; $\mathrm{AU}$, arbitrary unit; hsCRP, high-sensitivity C-reactive protein.

significant $(P>0.05)$. It is important to note that patients in the OSA group demonstrated a much wider distribution of values for all lipid parameters versus the control group. Figures illustrate quantification of lipid profiles by an ${ }^{1} \mathrm{H}$-MRS spectrum from the vastus lateralis muscle of a patient with severe OSA (Figure 1) and a control (Figure 2).

Table 2 also demonstrates the results of hsCRP levels, lipid profiles, biochemical analyses, and functional performance measurements for the two groups. Significantly higher hsCRP levels were observed in the severe OSA group (0.23 $\pm 0.14 \mathrm{mg} / \mathrm{dL})$ versus the control group $(0.12 \pm 0.06 \mathrm{mg} / \mathrm{dL})$ $(P<0.05)$. Patients with OSA also had significantly higher levels of insulin $(9.34 \pm 6.01 \mu \mathrm{U} / \mathrm{mL})$ and insulin resistance index (IR) (2.69 \pm 1.88$)$ compared with the controls $(P<0.05)$, but no significant differences were observed between the two groups in fasting glucose levels and lipid profiles. Additionally, patients with OSA demonstrated a significantly lower $\mathrm{VO}_{2 \text { peak }}$ $(P=0.038)$, and strength $(P<0.001)$ and endurance $(P=0.005)$ of knee extensors versus the control group.

The Spearman correlation coefficients of IMCL and EMCL, ratio of IMCL to EMCL, and various parameters for all participants are listed in Table 3. IMCL was negatively correlated with PSG, ODI, TspO2<90\% $(\rho=-0.35, P<0.05)$, arousal index, hsCRP, IR $(\rho=-0.40, P<0.05)$, glucose ( $\rho=-0.33, P<0.05)$, and insulin $(\rho=-0.36, P<0.05)$, and positively correlated with lowest $\mathrm{SpO}_{2}(\rho=0.33, P<0.01)$, $\mathrm{VO}_{2 \text { peak, }}$ strength, endurance, and daily energy expenditure.

\section{Discussion}

This study demonstrates that patients with OSA had significantly lower IMCL and EMCL values, as measured by ${ }^{1} \mathrm{H}$-MRS, compared with their age-, and BMI-matched controls without OSA. Compared with controls, patients with OSA also had significantly reduced functional performance and exhibited abnormal biochemical data, such as hsCRP, fasting insulin, and IR. It is suggested that both altered skeletal muscle and systemic metabolism are associated with OSA. Previous studies on skeletal muscle IMCL content in OSA populations are scarce and did not observe the correlation between measures of IMCL and IR in OSA. ${ }^{17,18}$ Our results enhance the understanding of the role of skeletal muscle metabolism in severe OSA.

The physiological function of fat in the muscle is to serve as a readily available intracellular source of energy during exercise. $^{32}$ This capacity to store fat inside the muscle may have conferred an evolutionary advantage to permit physical activity during cycles of feast and famine. ${ }^{32,33}$ The IMCL content is increased in the physically trained state to optimally match the fat oxidative capacity. However, nowadays the lower levels of physical activity combined with continuous availability of food in the population would negate the need for high IMCL content. In these conditions in which IMCLs are not being used for oxidation, IMCLs and their intermediates negatively affect insulin signaling and induce insulin resistance. ${ }^{33}$ Therefore, the preserved capacity to store fat in the muscle may have detrimental effects on insulin sensitivity. In this respect, the capacity to use IMCLs may be more influential than the magnitude of IMCL levels per se in determining the negative effects on insulin sensitivity.

Several studies have investigated the relationships between IMCL and insulin sensitivity. Our results were inconsistent with previous studies, indicating that increased content of IMCL is associated with a decreased whole-body and skeletal muscle insulin sensitivity in both patients with and without diabetes. ${ }^{11,34,35}$ Tamura et al examined the effects of diet and exercise on IMCL in patients with type II diabetes using ${ }^{1} \mathrm{H}-\mathrm{MRS} .{ }^{36}$ They demonstrated that IMCL decreased in parallel with improved peripheral insulin sensitivity. However, some studies did not demonstrate the relationship between IMCL and insulin resistance in non-obese 


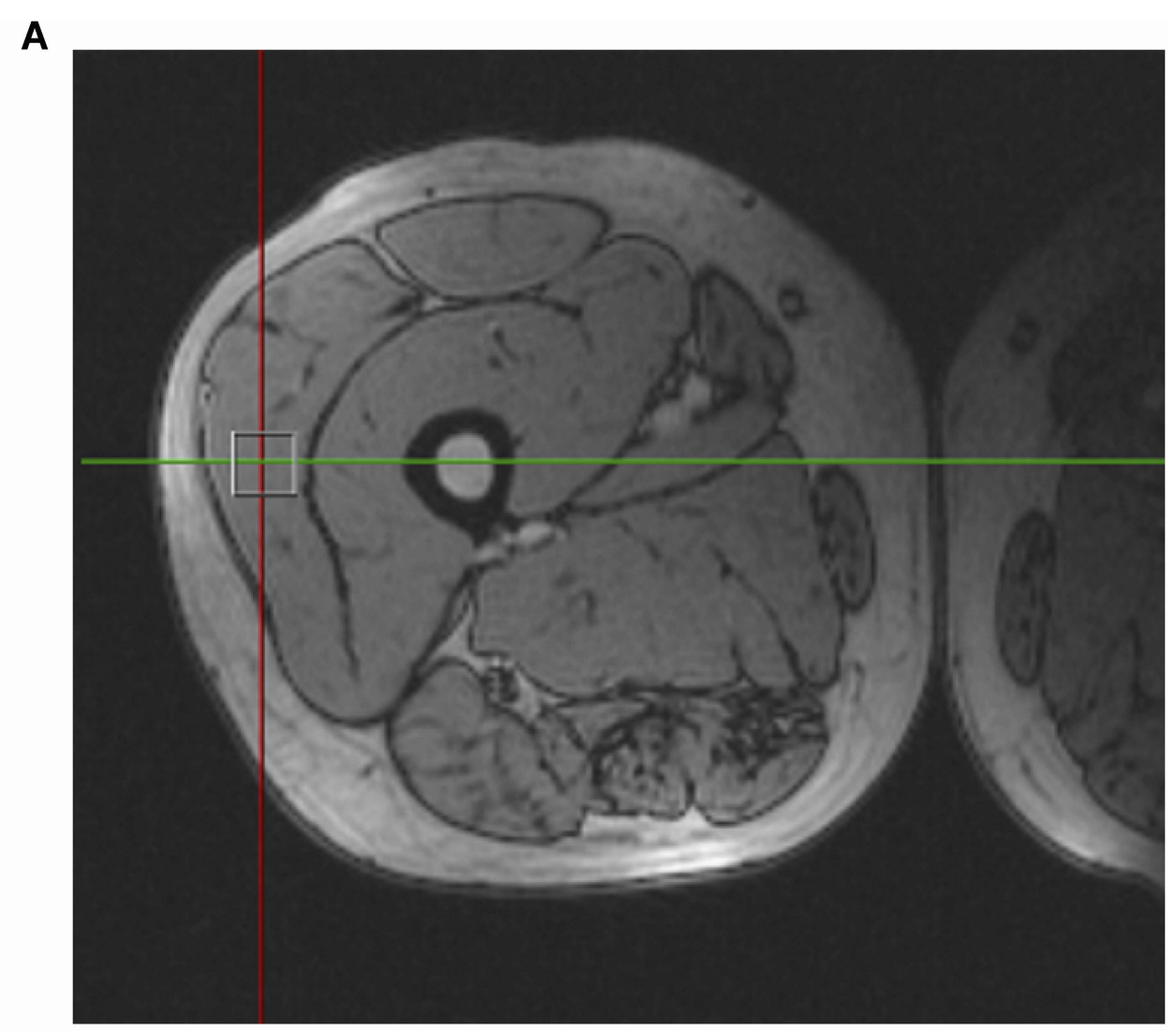

B

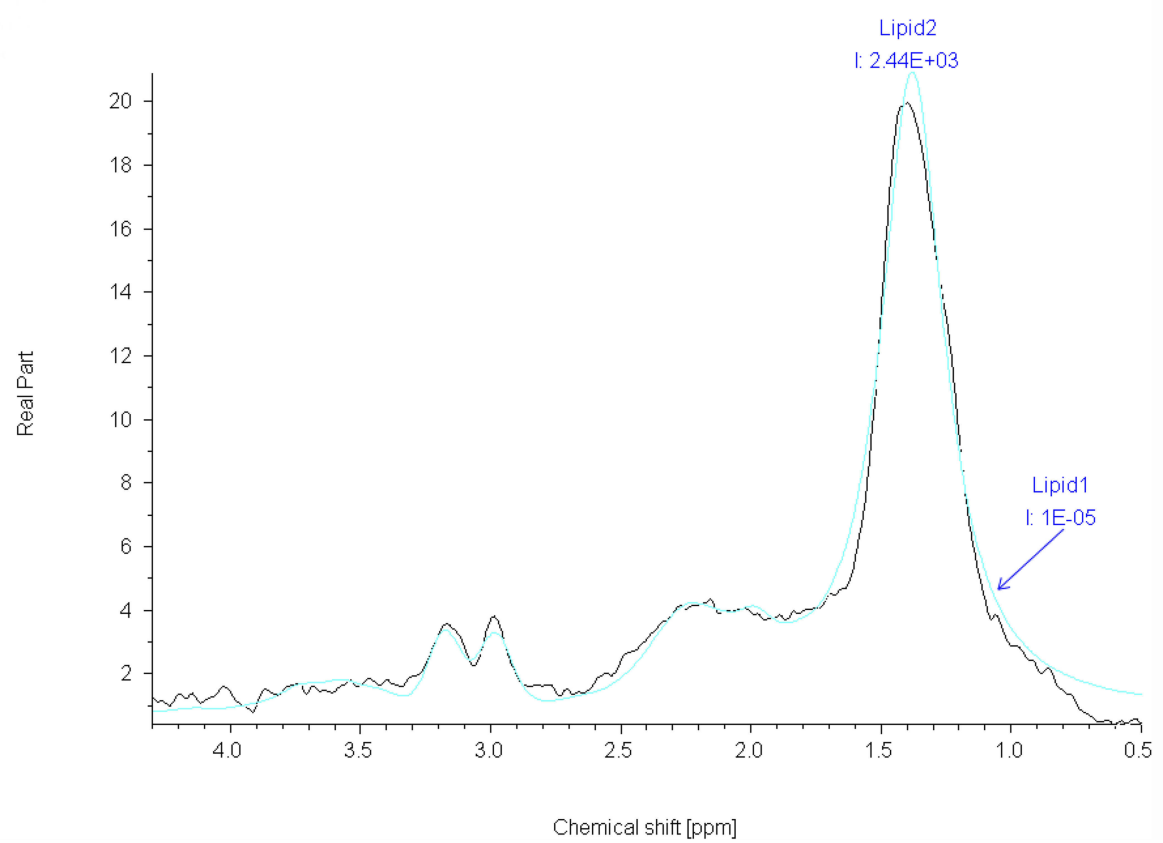

Figure I (A) 'H-MRS voxel placement from the vastus lateralis muscle of a patient with severe OSA. (B) The peak originating from the methylene protons of IMCLs of a patient with severe OSA by an 'H-MRS spectrum. 


\section{A}
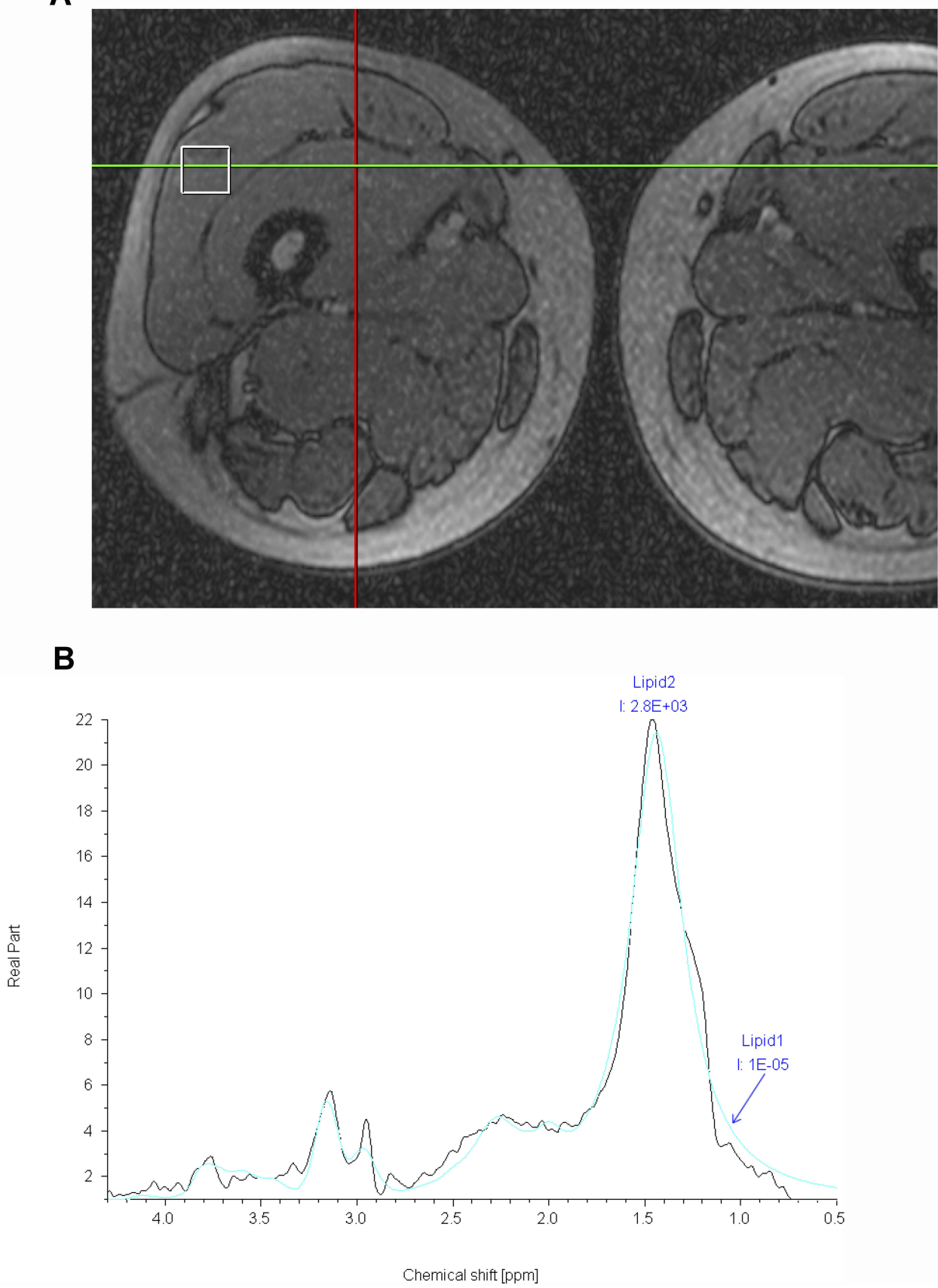

Figure 2 (A) 'H-MRS voxel placement from the vastus lateralis muscle of a control participant. (B) The peak originating from the methylene protons of IMCLs of a control participant by an 'H-MRS spectrum.

Indian patients with diabetes. ${ }^{12,13,37}$ Recent studies have highlighted ethnic differences in the determinants of the relationship between IMCL and insulin resistance. Both Ingram et $\mathrm{al}^{33}$ and Lawrence et $\mathrm{al}^{38}$ reported that IMCL relates to insulin sensitivity and adiposity in European Americans, but not in African Americans.

It is still unknown why African Americans and European Americans exhibit different relationships between
IMCL and insulin sensitivity. One possible explanation is that IMCL could be compartmentalized differently in different ethnicities. ${ }^{39}$ Another possible explanation is a difference in skeletal muscle fiber type between different ethnicities. Insulin resistance has been related to a higher IMCL content in type I (oxidative) muscle fibers, compared with type II (glycolytic) muscle fibers. ${ }^{40}$ It was reported that OSA would lead to a slow-to-fast muscle fiber-type 
Table 3 Correlation Coefficients Between Biochemistrical Data and Proton Magnetic Resonance Spectroscopy Results $(n=40)$

\begin{tabular}{|c|c|c|c|}
\hline & IMCL & EMCL & $\begin{array}{l}\text { IMCL/ } \\
\text { EMCL }\end{array}$ \\
\hline Apnea-hypopnea index (/hr) & -0.27 & -0.10 & 0.02 \\
\hline Oxygen desaturation index $(/ \mathrm{hr})$ & -0.27 & -0.09 & 0.05 \\
\hline $\mathrm{TSpO}_{2}<90 \%(\%)$ & $-0.35^{*}$ & -0.23 & 0.01 \\
\hline Arousal index $(/ \mathrm{hr})$ & -0.15 & -0.18 & 0.24 \\
\hline Sleep efficiency (\%) & 0.19 & $0.42 *$ & $-0.4 I^{*}$ \\
\hline Lowest $\mathrm{SpO}_{2}(\%)$ & $0.33^{*}$ & 0.18 & -0.04 \\
\hline Body mass index $\left(\mathrm{kg} / \mathrm{m}^{2}\right)$ & 0.24 & 0.14 & -0.13 \\
\hline Body fat $(\%)$ & -0.27 & 0.04 & -0.03 \\
\hline Fat-free mass (kg) & -0.07 & -0.11 & 0.01 \\
\hline $\mathrm{VO}_{2 \text { peak }}(\mathrm{mL} / \mathrm{kg} / \mathrm{min})$ & 0.19 & 0.12 & -0.04 \\
\hline Strength $(\mathrm{N}-\mathrm{m})$ & 0.25 & -0.03 & 0.19 \\
\hline Endurance $(\mathrm{N}-\mathrm{m})$ & 0.25 & 0.24 & -0.12 \\
\hline Energy expenditure $(\mathrm{kcal} / \mathrm{kg} /$ day $)$ & 0.14 & -0.02 & 0.13 \\
\hline Insulin resistance index & $-0.40 *$ & -0.25 & 0.04 \\
\hline Fasting glucose $(\mathrm{mg} / \mathrm{dL})$ & $-0.33 *$ & -0.30 & 0.17 \\
\hline Insulin $(\mu \mathrm{U} / \mathrm{mL})$ & $-0.36 *$ & -0.11 & -0.10 \\
\hline hsCRP (mg/dL) & -0.11 & -0.22 & -0.14 \\
\hline Total cholesterol (mg/dL) & -0.30 & -0.29 & 0.07 \\
\hline Triglyceride (mg/dL) & 0.01 & -0.13 & 0.11 \\
\hline High-density lipoprotein ( $\mathrm{mg} / \mathrm{dL})$ & -0.24 & -0.27 & 0.17 \\
\hline Low-density lipoprotein (mg/dL) & -0.22 & -0.11 & -0.01 \\
\hline
\end{tabular}

Notes: *Indicates statistically significant as $P<0.05$. $\mathrm{TSPO}_{2}<90 \%$ is the cumulative time with nocturnal pulse oximetric saturation lower than $90 \%$.

Abbreviations: IMCL, intramyocellular lipid contents; EMCL, extramyocellular lipid contents; hsCRP, high-sensitivity C-reactive protein.

transformation. Thus, it is plausible that more type II fibers within the vastus lateralis muscle in the OSA group than in the control group might contribute to this phenomenon.

Because of the unique features of intermittent hypoxia and their chronic, low-grade inflammatory status, patients with OSA were considered at a high risk for developing metabolic syndrome. It was speculated that elevated IMCL levels and IR would be present in patients with OSA. Trenell et a $1{ }^{17}$ reported no change in IMCL content and insulin sensitivity scores after 12-week treatment of CPAP in patients with OSA. Our study demonstrated that patients with severe OSA exhibited a higher insulin level and IR, but there was a reduction in both IMCL and EMCL. Therefore, a significantly negative correlation between IMCL and both $\mathrm{TSpO}_{2}$ and IR was reported. In addition to ethnic differences and muscle fiber-type variations, insufficient energy supply in OSA patients is another possible mechanism, based on the reduced $\mathrm{VO}_{2 \text { peak }}$ observed in OSA patients. As previously mentioned, the relationships between IMCL, glucose, and insulin are complex and depend on several factors.
More research is required to further investigate these relationships.

${ }^{1} \mathrm{H}$-MRS of human skeletal muscle has become more commonly investigated in clinical practice, primarily due to the relationship between IMCL levels and insulin sensitivity. ${ }^{41}$ Several longitudinal studies have highlighted the utility of ${ }^{1} \mathrm{H}$-MRS to quantify IMCL over time. ${ }^{1} \mathrm{H}-\mathrm{MRS}$ has been validated in humans and the error of this method is reported to be as low as approximately $6 \%$. ${ }^{42,43}$ The test-retest reliability of ${ }^{1} \mathrm{H}-\mathrm{MRS}$ IMCL measurements in humans is acceptable, and changes in IMCL greater than $15 \%$ could be detected between ${ }^{1} \mathrm{H}-\mathrm{MRS}$ measurements. ${ }^{43}$ However, there are limitations of using ${ }^{1}$ H-MRS to quantify IMCL. First, IMCL is typically expressed in relative terms as the ratio between the signal peak areas in the respective spectra, resulting from IMCL and creatinine or water. Secondly, the IMCL signal highly depends on the orientation of the muscle fibers so that only the lipid of selected voxels within certain muscle groups can be reliably measured. ${ }^{42}$ Therefore, the leg must be carefully positioned to ensure muscle fibers are correctly oriented. Also, the regions of interest containing voxels must be carefully selected to avoid contamination.

There are several study limitations that should be considered. First, given the cross-sectional nature of this study, any causal relationship between these constructs remains to be determined. Future longitudinal studies are required to verify the sequential changes and relationships reported in this study. Second, evidence showed that IMCL content was not stable and might change over time. Factors such as diet, obesity state, and level of physical activity have profound effects on IMCL. ${ }^{10}$ As the groups were matched for the anthropometric parameters and no significant difference of daily energy expenditure was found between two groups, the difference in IMCL could not be attributed to these factors. However, future studies should control the dietary factors, as these were not considered in the present study. Third, our participants were limited to middle-aged men. Thus, the results could not be extrapolated to women or other ethnicities.

\section{Conclusion}

The present study demonstrated that patients with severe OSA exhibited a reduction in IMCL and EMCL, poor aerobic and muscular performance, and impaired biochemical data, including glucose and insulin levels and lipid profiles. It appeared that both systemic and skeletal muscle metabolism dysfunction occurs in the OSA group, but not 
in the control subjects. Additionally, IMCL positively correlated with aerobic capacity and muscular performance, but negatively correlated with AHI and IR. For patients with OSA that did not have obesity, lower IMCL might indicate insufficient energy storage in muscles compared with their counterparts. However, additional large-scale clinical trials are required to further explore the complex mechanism between OSA, muscle metabolism, and insulin action.

\section{Data Sharing Statement}

The authors do not intend to share individual deidentified participant data.

\section{Acknowledgment}

The authors thank the National Science Council (Taiwan) for financial support (NSC 96-2314-B-002-022-MY3). The authors also thank Professor Yunn-Jy Chen from Division of Prosthodontics, Department of Dentistry, National Taiwan University Hospital and Graduate Institute of Clinical Dentistry, School of Dentistry, College of Medicine, National Taiwan University, for his great contribution to this research.

\section{Disclosure}

None of the authors has a financial relationship with a commercial entity that has an interest in the subject of this manuscript. The authors report no conflicts of interest in this work.

\section{References}

1. American Academy of Sleep Medicine. Sleep-related breathing disorders in adults: recommendations for syndrome definition and measurement techniques in clinical research. The Report of an American Academy of Sleep Medicine Task Force. Sleep. 1999;22(5):667-689. doi:10.1093/sleep/22.5.667

2. Ip MS, Lam B, Ng MM, et al. Obstructive sleep apnea is independently associated with insulin resistance. Am J Respir Crit Care Med. 2002;165(5):670-676. doi:10.1164/ajrccm.165.5.2103001

3. Punjabi NM, Shahar E, Redline S, et al. Sleep Heart Health Study I. Sleep-disordered breathing, glucose intolerance, and insulin resistance: the Sleep Heart Health Study. Am J Epidemiol. 2004;160(6):521-530. doi:10.1093/aje/kwh261

4. Kelley DE. Skeletal muscle fat oxidation: timing and flexibility are everything. J Clin Invest. 2005;115(7):1699-1702. doi:10.1172/JCI25758

5. Petersen KF, Dufour S, Befroy D, et al. Impaired mitochondrial activity in the insulin resistant offspring of patients with type 2 diabetes. $N$ Engl $J$ Med. 2004;350(7):664-671. doi:10.1056/NEJMoa031314

6. Pastoris O, Dossena M, Foppa P, et al. Modification by chronic intermittent hypoxia and drug treatment on skeletal muscle metabolism. Neurochem Res. 1995;20(2):143-150. doi:10.1007/BF00970538

7. Bonanni E, Pasquali L, Manca ML, et al. Lactate production and catecholamine profile during aerobic exercise in normotensive OSAS patients. Sleep Med. 2004;5(2):137-145. doi:10.1016/j.sleep.2003.08.009
8. Edward Shifflett D, Walker EW, Gregg JM, et al. Effects of short-term PAP treatment on endurance exercise performance in obstructive sleep apnea patients. Sleep Med. 2001;2(2):145-151. doi:10.1016/S1389-9457(00)00070-8

9. Dubé J, Goodpaster BH. Assessment of intramuscular triglycerides: contribution to metabolic abnormalities. Curr Opin Clin Nutr Metab Care. 2006;9(5):553-559. doi:10.1097/01.mco.0000241664.38385.12

10. Machann J, Stefan N, Schick F. ${ }^{1}$ H MR spectroscopy of skeletal muscle, liver and bone marrow. Eur J Radiol. 2008;67(2):275-284. doi:10.1016/j.ejrad.2008.02.032

11. Machann J, Haring H, Schick F, et al. Intramyocellular lipids and insulin resistance. Diabetes Obes Metab. 2004;6(4):239-248. doi:10. 1111/dom.2004.6.issue-4

12. Misra A, Sinha S, Kumar M, et al. Proton magnetic resonance spectroscopy study of soleus muscle in non-obese healthy and type 2 diabetic Asian Northern Indian males: high intramyocellular lipid content correlates with excess body fat and abdominal obesity. Diabetic Med. 2003;20(5):361-367. doi:10.1046/j.1464-5491.2003.0 0932.x

13. Forouhi NG, Jenkinson G, Thomas EL, et al. Relation of triglyceride stores in skeletal muscle cells to central obesity and insulin sensitivity in European and South Asian men. Diabetologia. 1999;42(8):93 2-935. doi:10.1007/s001250051250

14. Boesch C, Machann J, Vermathen P, et al. Role of proton MR for the study of muscle lipid metabolism. NMR Biomed. 2006;19(7):96 8-988. doi:10.1002/(ISSN)1099-1492

15. Schick F, Eismann B, Jung WI, et al. Comparison of localized proton NMR signals of skeletal muscle and fat tissue in vivo: two lipid compartments in muscle tissue. Magn Reson Med. 1993;29(2):15 8-167. doi:10.1002/(ISSN)1522-2594

16. Petersen KF, Shulman GI. Pathogenesis of skeletal muscle insulin resistance in type 2 diabetes mellitus. Am J Cardiol. 2002;90(5A): G11-G18. doi:10.1016/S0002-9149(02)02554-7

17. Trenell MI, Ward JA, Yee BJ, et al. Influence of constant positive airway pressure therapy on lipid storage, muscles metabolism and insulin action in obese patients with severe obstructive sleep apnoea syndrome. Diabetes Obes Metab. 2007;9(5):679-687. doi:10.1111/ j.1463-1326.2006.00649.x

18. Canapari CA, Hoppin AG, Kinane TB, et al. Relationship between sleep apnea, fat distribution, and insulin resistance in obese children. J Clin Sleep Med. 2011;7(3):268-273. doi:10.5664/JCSM.1068

19. Chien MY, Lee PL, Tsai YF, et al. C-reactive protein and heart rate recovery in middle-aged men with severe obstructive sleep apnea. Sleep Breath. 2012;16(3):629-637. doi:10.1007/s11325-01 1-0549-2

20. Chien MY, Wu YT, Lee PL, et al. Inspiratory muscle dysfunction in patients with severe obstructive sleep apnea. Eur Respir J. 2010;35 (2):373-380. doi:10.1183/09031936.00190208

21. John MW. A new method for measuring daytime sleepiness: the Epworth sleepiness scale. Sleep. 1991;14(6):540-545. doi:10.1093/ sleep/14.6.540

22. Roberts WL, Moulton L, Law TC, et al. Evaluation of nine automated high-sensitivity $\mathrm{C}$-reactive protein methods: implications for clinical and epidemiological applications. Part 2. Clin Chem. 2001;47 (3):418-425.

23. Vikram NK, Pandey RM, Misra A, et al. Non-obese (body mass index $<25 \mathrm{~kg} / \mathrm{m} 2)$ Asian Indians with normal waist circumference have high cardiovascular risk. Nutrition. 2003;19(6):503-509. doi:10. 1016/s0899-9007(02)01083-3

24. Friedewald WT, Levy RI, Fredrickson DS. Estimation of the concentration of low-density lipoprotein cholesterol in plasma, without use of the preparative ultracentrifuge. Clin Chem. 1972;18(6):499-502.

25. Matthews DR, Hosker JP, Rudenski AS, et al. Homeostasis model assessment: insulin resistance and beta-cell function from fasting plasma glucose and insulin concentrations in man. Diabetologia. 1985;28(7):412-419. doi:10.1007/BF00280883 
26. Pollock ML, Graves JE, Mahar MT. Reliability and validity of bioelectrical impedance in determining body composition. J Appl Physiol. 1988;64(2):529-534. doi:10.1152/jappl.1988.64. 2.529

27. Thomson R, Brinkworth GD, Buckley JD, et al. Good agreement between bioelectrical impedance and dual-energy X-ray absorptiometry for estimating changes in body composition during weight loss in overweight young women. Clin Nutr. 2007;26(6):771-777. doi:10. 1016/j.clnu.2007.08.003

28. Wilcock A, Maddocks M, Lewis M, et al. Use of a Cybex NORM dynamometer to assess muscle function in patients with thoracic cancer. BMC Palliat Care. 2008;7:3. doi:10.1186/1472-684X-7-3

29. American College of Sports Medicine. ACSM's Guidelines for Exercise Testing and Prescription. 7th ed. Philadelphia, PA: Lippincott Williams \& Wilkins; 2006.

30. Hwang JH, Pan JW, Heydari S, et al. Regional differences in intramyocellular lipids in human observed by in vivo ${ }^{1} \mathrm{H}-\mathrm{MRS}$ spectroscopic imaging. J Appl Physiol. 2001;90(4):1267-1274. doi:10.1152/ jappl.2001.90.4.1267

31. Pickering TG, Hall JE, Appel LJ, et al. Recommendations for blood pressure measurement in humans and experimental animals. Part I: blood pressure measurement in humans: statement for professionals from the subcommittee of professional and public education of the American Heart Association Council on High Blood Pressure Research. Circulation. 2005;111(5):697-716. doi:10.1161/01.CIR.00 00154900.76284.F6

32. Schrauwen-Hinderling VB, Hesselink MKC, Schrauwen P, et al. Intramyocellular lipid content in human skeletal muscle. Obesity. 2006;14(3):357-367. doi:10.1038/oby.2006.47

33. Ingram KH, Lara-Castro C, Gower BA. Intramyocellular lipid and insulin resistance: differential relationships in European and African Americans. Obesity. 2011;19(7):1469-1475. doi:10.1038/ oby. 2011.45

34. Jacob S, Machann J, Rett K, et al. Association of increased intramyocellular lipid content with insulin resistance in lean nondiabetic offspring of type 2 diabetic subjects. Diabetes. 1999;48(5):1113-11 19. doi: $10.2337 /$ diabetes.48.5.1113
35. Virkamaski A, Korsheninnikova E, Seppala-Lindroos A, et al. Intramyocellular lipid associated with resistance to in vivo insulin actions on glucose uptake, antilipolysis, and early insulin signaling pathways in human skeletal muscle. Diabetes. 2001;50(10):2 337-2343. doi:10.2337/diabetes.50.10.2337

36. Tamura Y, Tanaka Y, Sato F, et al. Effects of diet and exercise on muscle and liver intracellular lipid contents and insulin sensitivity in type 2 diabetic patients. J Clin Endocrinol Metab. 2005;90 (6):3191-3196. doi:10.1210/jc.2004-1959

37. Sinha R, Rathi M, Misra A, et al. Subclinical inflammation and soleus muscle intramyocellular lipids in healthy Asian Indian males. Clin Endocrinol. 2005;63(3):350-355. doi:10.1111/j.1365-2265.2005.02353.x

38. Lawrence JC, Newcomer BR, Buchthal SD, et al. Relationship of intramyocellular lipid to insulin sensitivity may differ with ethnicity in healthy girls and women. Obesity (Silver Spring). 2011;19 (1):43-48. doi:10.1038/oby.2010.148

39. Nielsen J, Mogensen M, Vind BF, et al. Increased subsarcolemmal lipids in type 2 diabetes: effect of training on localization of lipids, mitochondria, and glycogen in sedentary human skeletal muscle. Am J Physiol Endocrinol Metab. 2010;298(3):E706-E713. doi:10.1152/ ajpendo.00692.2009

40. Coen PM, Dube JJ, Amati F, et al. Insulin resistance is associated with higher intramyocellular triglycerides in type I but not type II myocytes concomitant with higher ceramide content. Diabetes. 2010;59(1):80-88. doi:10.2337/db09-0988

41. Perseghin G, Scifo P, De Cobelli F, et al. Intramyocellular triglyceride content is a determinant of in vivo insulin resistance in humans: a ${ }^{1} \mathrm{H}-{ }^{13} \mathrm{C}$ nuclear magnetic resonance spectroscopy assessment in offspring of type 2 diabetic parents. Diabetes. 1999;48(8):1600-1606. doi:10.2337/diabetes.48.8.1600

42. Boesch C, Slotboom J, Hoppeler H, et al. In vivo determination of intra-myocellular lipids in human muscle by means of localized ${ }^{1}$ H-MR-spectroscopy. Magn Reson Med. 1997;37(2):484-493. doi:10.1002/mrm. 1910370403

43. Larson-Meyer DE, Smith SR, Heilbronn LK, et al. Muscle-associated triglyceride measured by computed tomography and magnetic resonance spectroscopy. Obesity (Silver Spring). 2006;14(1):73-87. doi:10.1038/oby.2006.10
Nature and Science of Sleep

\section{Publish your work in this journal}

Nature and Science of Sleep is an international, peer-reviewed, open access journal covering all aspects of sleep science and sleep medicine, including the neurophysiology and functions of sleep, the genetics of sleep, sleep and society, biological rhythms, dreaming, sleep disorders and therapy, and strategies to optimize healthy sleep.
The manuscript management system is completely online and includes a very quick and fair peer-review system, which is all easy to use. Visit http://www.dovepress.com/testimonials.php to read real quotes from published authors. 